\title{
ENDOTHELIAL CELL VACUOLATION IN THE CORNEA*
}

\author{
BY \\ JOHN SPEAKMAN† \\ Department of Pathology, Institute of Ophthalmology, University of London
}

IN a recent study of the drainage channels through the trabecular meshwork (Speakman, 1959), attention was directed to a remarkable degree of vacuolation in the endothelial cells lining the trabecular wall of Schlemm's canal which has also been described in an electron microscopic study of this region by Garron, Feeney, Hogan, and McEwen (1958). Since endothelial cells of the cornea are in direct continuity with those of the meshwork and are more readily studied, a detailed examination of these cells has been made in an attempt to elucidate further the significance of the vacuolation present in the cells lining the inner wall of Schlemm's canal.

In the literature, the corneal endothelium has been described as a mosaic of hexagonal cells, and reference has recently been made to an abundant cement substance located between the cells, which can be seen with the phase microscope or with special stains (Wolter, 1957; Vrabec, 1958; Chi, Teng, and Katzin, 1958).

Descriptions of the patterns formed by the so-called cement substance vary greatly from one paper to another and also, according to Vrabec, in different regions of the same eye.

It will be shown that these findings are not a result of alterations in the distribution of cement substance but are due to rapid post-mortem vacuolation which produces a variable distortion of the cells.

\section{Materials}

Human eyes were studied which had been removed because of suspected intraocular tumours and fixed in the usual way by dropping the unopened eye into 10 per cent. formalin solution. Comparisons were made with cat and rabbit eyes which were placed opened and unopened in a variety of fixatives. The phase microscope was employed to follow the development of vacuolation in unfixed endothelium immersed in warm Tyrode's solution, and Indian ink in gelatine was run over the surface of the corneal endothelium to outline the cell boundaries and to demonstrate the vacuoles.

\section{Observations}

\section{(1) Vacuolation in the Endothelium of the Trabecular Meshwork}

In a previous paper (Speakman, 1959) reference was made to fine vacuolation which can be recognized occasionally in the cytoplasm of the uveal meshwork and frequently in the cytoplasm surrounding the corneo-scleral fibres. In flat preparations much larger vacuoles can be seen in the

* Received for publication November 11, 1958.

+ Wernher Pigott Research Fellow, Department of Ophthalmology, University of Toronto. 
endothelial cells lining the trabecular wall of the canal, which vary in diameter from $2 \mu$ to $5 \mu$ and often appear at each end of the fusiform nuclei (Fig. 1). Sections through the inner wall of the canal show the vacuolated cells cut in a variety of planes (Fig. $2 a, b$ ). They are a constant feature of the inner wall and have been seen in numerous flat preparations and sections of this region in both premature and adult eyes.

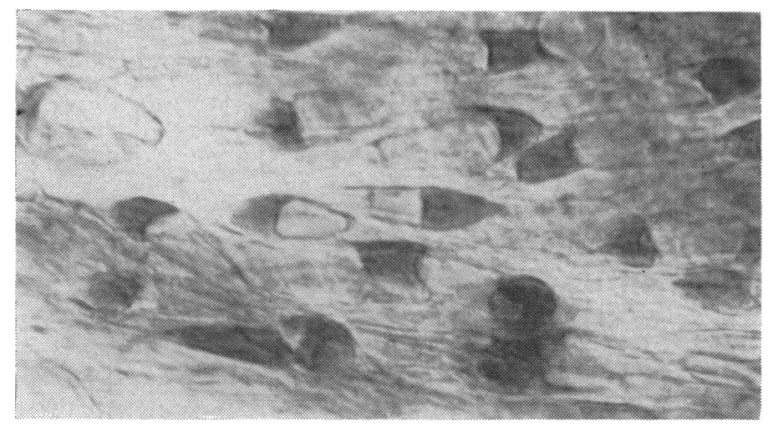

Fig. 1.-Flat preparation of trabecular wall of Schlemm's canal, showing vacuoles in endothelial cells. Human. Rinehart AbulHaj stain. $\times 950$.

(Figs 1 to 6: delayed formalin fixation).

$(2 a)$
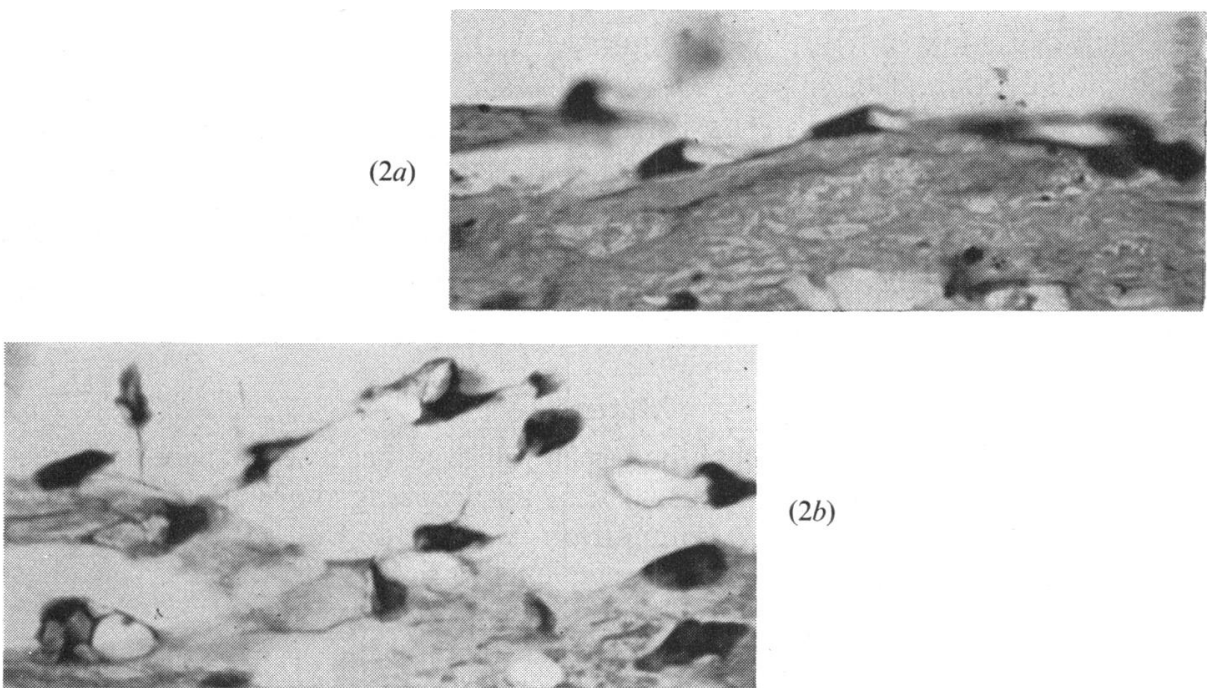

$(2 b)$

FIG. $2(a$ and $b$ ).-Sections through vacuolated cells on wall of Schlemm's canal. Human. Mallory stain. $\times 950$.

\section{(2) Vacuolation in the Corneal Endothelium}

(a) Delayed Fixation.-Paraffin sections of human and rabbit corneae from unopened eyes fixed in 10 per cent. formalin show a similar marked degree of vacuolation, which is more conspicuous in the cornea because the cells are packed more closely together (Fig. 3, opposite). Flat preparations of human eyes show how abruptly the gross vacuolation stops at Schwalbe's line in the premature baby (Fig. 4, opposite), and in the adult large vacuoles can be seen filling the cells and displacing the nuclei (Fig. 5, opposite). The 
Fig. 3.-Section through vacuolated corneal endothelium. Rabbit. Haematoxylin and eosin. $\times 950$.
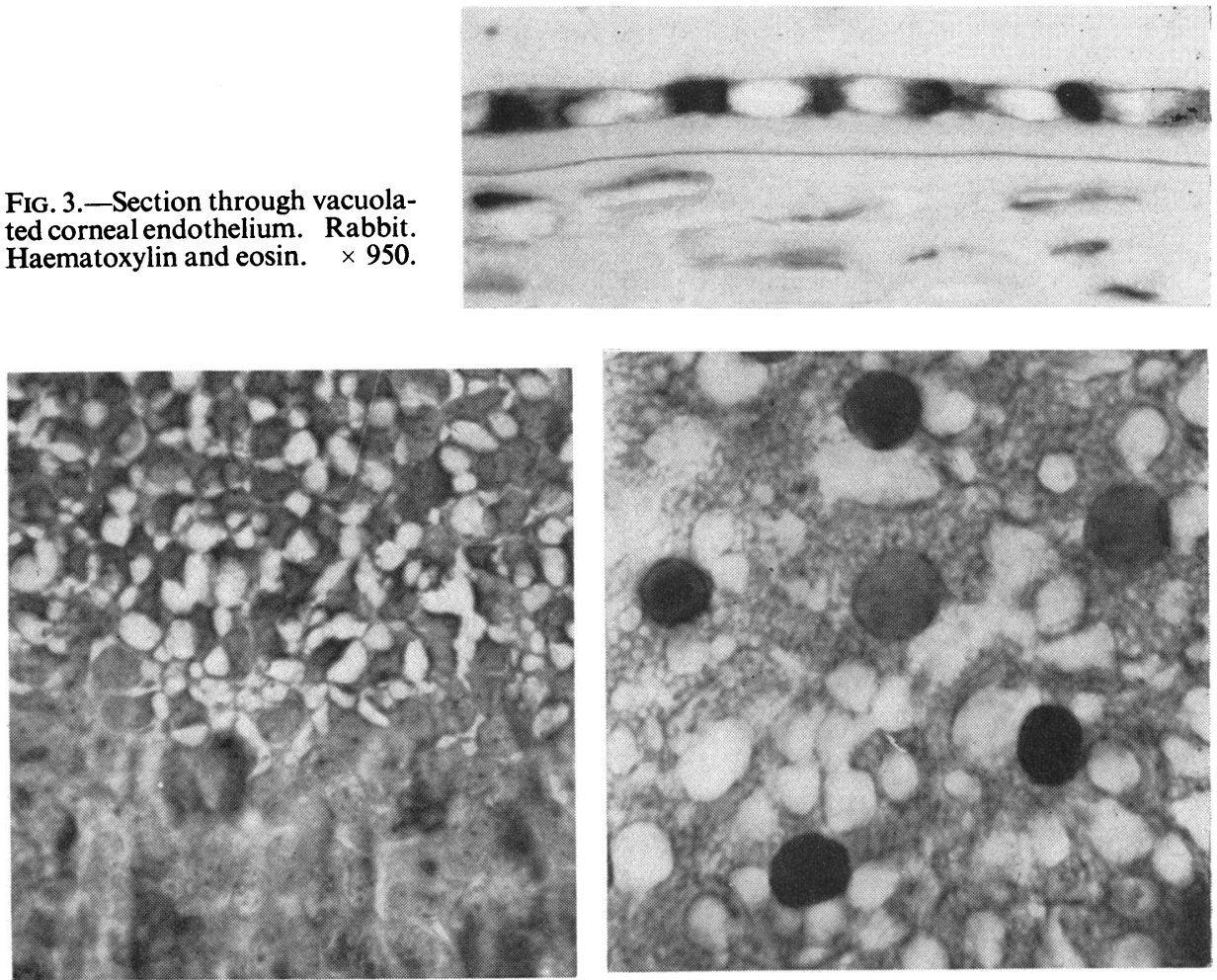

Fig. 4.-Flat preparation, showing abrupt transition from vacuolated to normal endothelium at Schwalbe's line. Premature human. Polychrome methylene blue stain. $\times 380$.

FIG. 5.-Flat preparation, showing vacuoles in corneal endothelium. Human. Rinehart Abul-Haj stain. $\times 950$.

Fig. 6.-Flat preparation of corneal endothelium treated with Indian ink to outline large vacuoles and cell boundaries. Human. $\times 950$.

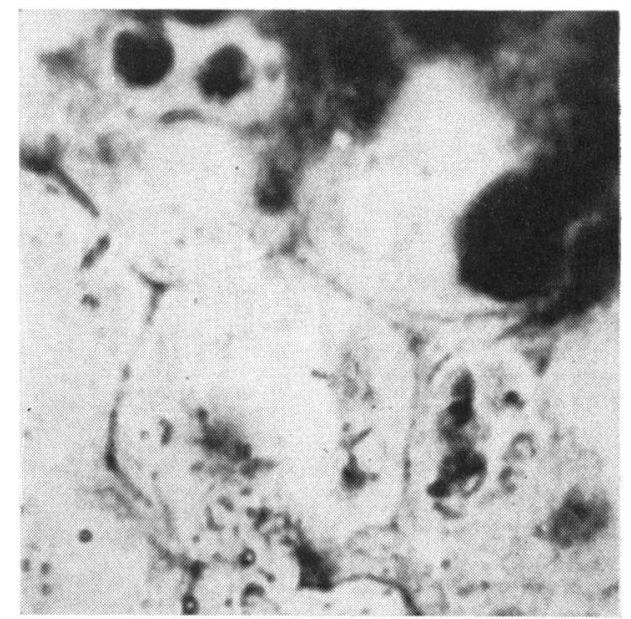

cytoplasm which forms the walls of the larger spaces is itself riddled with fine vacuoles.

The phase microscope shows the large vacuoles distorting the cells, but there is no evidence of any cement substance uniting the cells and a hexagonal pattern cannot be recognized. However, if Indian ink in gelatine (Fig. 6) 
is run over the surface of the endothelium in small quantities and allowed to harden, it forms large globular masses inside many of the vacuoles which open to the surface, and in depressions at the cell boundaries forms thin black lines which have a hexagonal pattern. Some of the pale areas within the cells are due to nuclei and others to vacuoles which did not open to the surface.

The distortion of the cytoplasm produced by the vacuoles shows that the customary procedure of placing intact eyes in fixative is not adequate for preserving the normal appearance of the corneal endothelium.

(b) Rapid Fixation.-It was felt that, if the vacuolation within the endothelial cells was due to post-mortem change, more rapid fixation techniques might prevent its occurrence. Corneae were therefore removed from the eyes of anaesthetized animals and placed immediately into various fixatives before examination. Rabbit corneal endothelium was removed in this way, and portions were fixed in formalin and others in osmic acid. Under lowpower magnification with the phase microscope the formalin-fixed specimens appeared homogeneous, but under high-power magnification numerous fine vacuoles were seen throughout the cytoplasm. These appeared to concentrate and coalesce at the periphery of the cell, where occasionally clear channels formed, outlining the cell boundary. When osmic acid was used as fixative the cytoplasm appeared uniformly granular and no definite vacuoles were seen (Fig. 7).

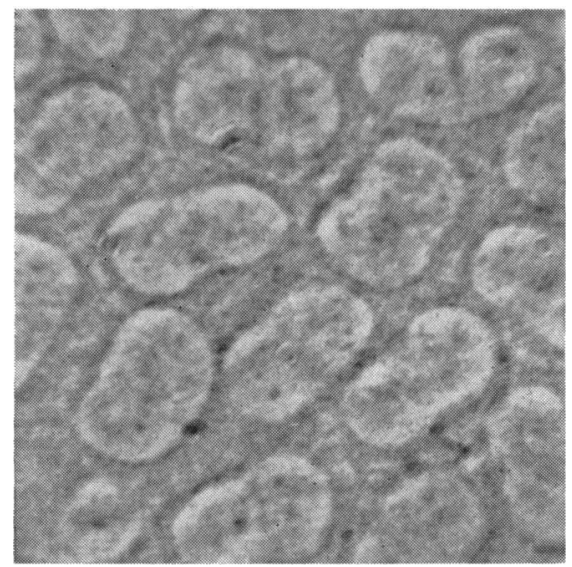

FIG. 7.-Flat preparation of corneal endothelium rapidly fixed with osmic acid, showing absence of vacuoles. Rabbit. Phase $\times 950$.

In the cat endothelium, rapidly fixed in formalin and examined under lowpower magnification by phase microscopy, a well-defined clear zone could be seen at the periphery of the cells, forming a hexagonal pattern. Under high magnification the clear zone was seen to consist of rows of small vacuoles lying side by side or superimposed upon each other (Fig. 8a). Some of the vacuoles appeared to have coalesced and others were located in the centre of the cells. In specimens stained with polychrome methylene blue the larger 

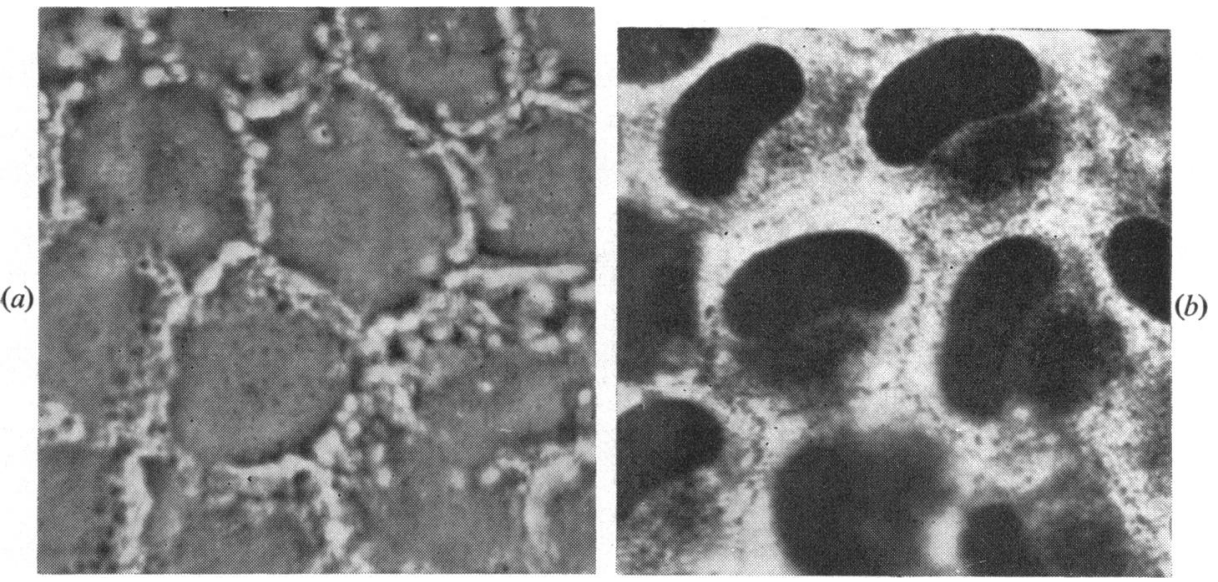

FIG. 8.-Flat preparation of corneal endothelium rapidly fixed in formalin, showing (a) rows of vacuoles at the cell periphery, $(b)$ argyrophilic granules in cytoplasm and at cell boundary. Cat $(a)$ Phase $\times 950 ;(b)$ Bielschowsky stain. $\times 950$.

vacuoles could still be recognized in rows at the periphery of the cells. In silver-stained preparations argyrophilic granules were present in the cytoplasm and precipitated stain formed a thin line at the cell boundary (Fig. 8b).

In Carnoy-fixed tissue, vacuoles were not recognizable but numerous cracks and fissures appeared which were thought to result from shrinkage produced by the fixative.

(c) Examination of Fresh Preparation.-In order to follow the development of vacuolation a fresh preparation of corneal endothelium from the rabbit was placed in warm Tyrode's solution and studied with the phase microscope. Initially a clear zone was seen at the periphery of each cell comparable to that seen in the cat endothelium fixed rapidly in formalin (Fig. 9a, overleaf).

At the end of 11 minutes this pattern disappeared and a number of large vacuoles formed within the cell distorting the normal cytoplasmic pattern (Fig. 9b, overleaf).

This stage was comparable to the appearance of corneal endothelium removed from eyes where there had been a delay in fixation. Finally after 72 minutes many of the cells ruptured and the nuclei disappeared, leaving a hexagonal pattern of dark lines formed by cytoplasmic granules adherent to each other at the periphery of the cell. Other cells retained their nuclei and contained large intercommunicating vacuoles (Fig. 9c, overleaf).

This stage resembled the pictures of cement substance shown in recent publications by Chi and others (1958) and Vrabec (1958) whose vitallystained preparations of corneal endothelium would inevitably have undergone extensive post-mortem change before fixation (Fig. 10, overleaf).

\section{Discussion}

There is a striking similarity between the vacuolation which develops in 


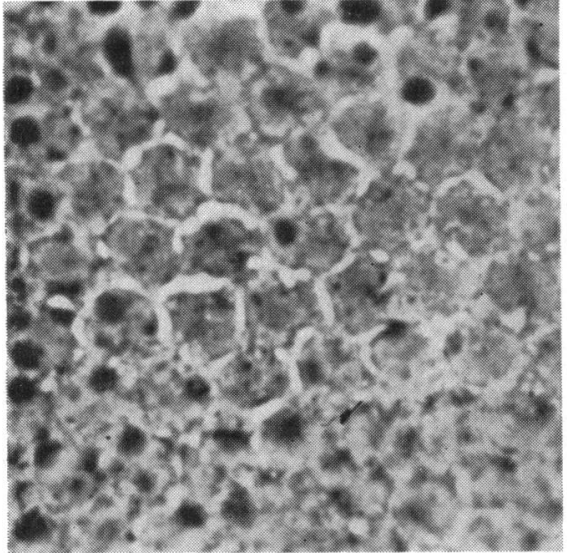

$(9 a)$

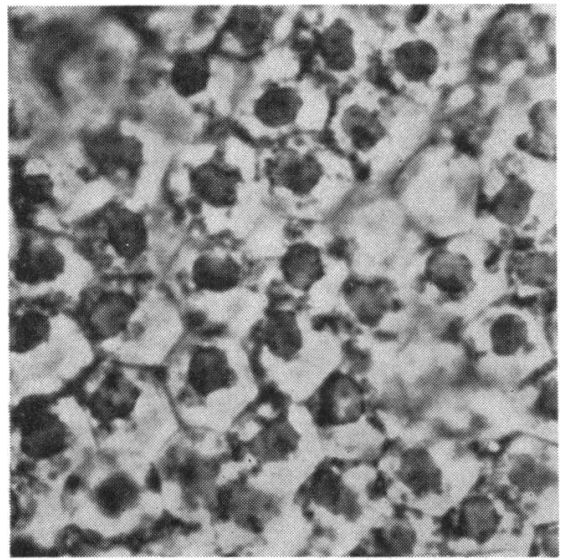

(9c)

Fig. 9.-Vacuolation developing in unfixed corneal endothelium mounted in warm Tyrode's solution.

(a) Initial appearance; (b) after 11 minutes; (c) after 72 minutes. Rabbit. Phase $x$ 400.

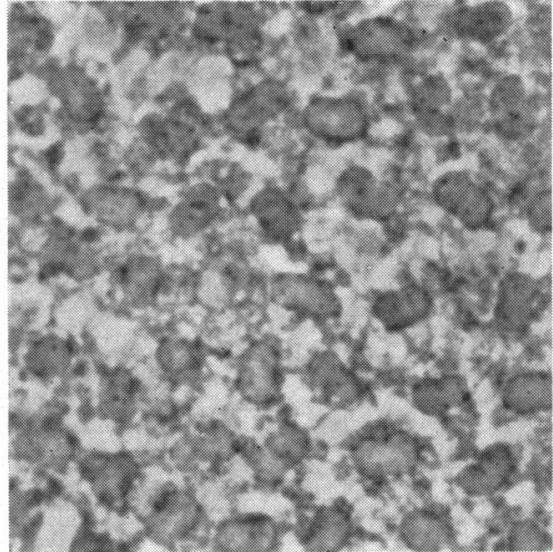

$(9 b)$

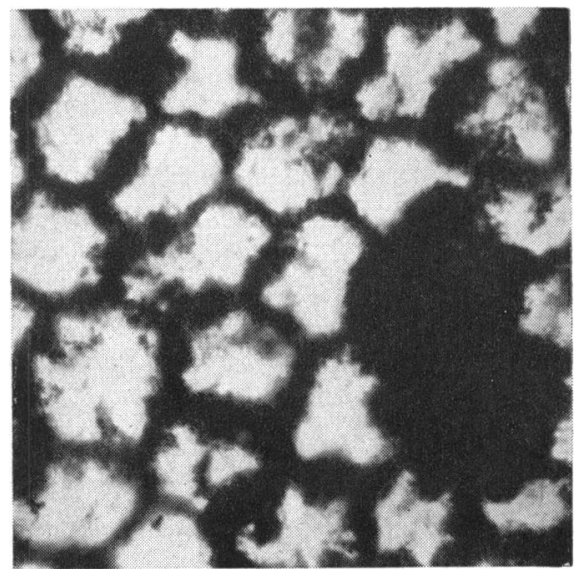

(10)

FIG. 10.-Argyrophilic cytoplasmic granules forming a hexagonal pattern at cell boundaries of corneal endothelium. Some cells have not burst, and in others clumps of granules remain attached to Descemet's membrane at the centre of the cell. Cat. McGovern stain. $\times 640$.

the endothelium lining the trabecular wall of Schlemm's canal and the endothelium covering the back of the cornea. The large vacuoles in the corneal endothelium stop at Schwalbe's line and in the endothelium lining Schlemm's canal they are largely confined to the inner trabecular wall. Is there an explanation for this puzzling distribution? The transparency of the corneal stroma is dependent on an intact layer of endothelium lining Descemet's membrane. When this layer is interrupted there is immediate clouding of the stroma due to the imbibition of fluid by corneal mucoid. The corneal endothelium, therefore, is essential in preventing the accumulation of excess 
fluid in the stroma. In flowing through the trabecular meshwork, aqueous must pass through very narrow channels which are particularly attenuated near Schlemm's canal. It is obvious that swelling of the trabecular fibres in this region (which, like the corneal stroma, consists of collagen fibrils in a mucopolysaccharide matrix) would seriously impede the outflow of aqueous. It seems reasonable therefore to suppose that the vacuolated cells on the wall of the canal are important in preventing swelling of the underlying fibres of the trabecular meshwork. Unfortunately, it is not known whether the trabecular fibres adjacent to the canal swell when the endothelium is selectively damaged, nor is it clear why the gross vacuolation should be confined to the canal wall. If the degree of vacuolation indicates the watertransporting capabilities of the cell it may be that the mucopolysaccharide in the fibres adjacent to the canal wall has an increased affinity for water not shared by the remainder of the meshwork.

It would be of interest to prevent the changes occurring in the trabecular endothelium by devising a rapid fixation technique and using the corneal endothelium as a control. Garron and others (1958) found vacuoles in the lining of Schlemm's canal in eyes which were perfused with fixative shortly after the death of the patient, and we have seen vacuolated cells lining the collector channels of the cat even though the eyes were opened and placed in formalin within minutes of the death of the animal. This procedure checked but did not completely prevent the development of vacuolation in the corneal endothelium, and it is possible that the less accessible cells of the collector channels were not reached quickly enough by the fixative.

It is interesting that Nuel and Cornil (1890) appreciated the rapid postmortem changes which occur in the corneal endothelium and pointed out that many writers had described the degenerate state as normal. They thought the changes were due to a process of cytoplasmic constriction and alteration of intra-cellular fibrils; they did not realize that the degeneration is the result of a progressive enlargement of intra-cellular vacuoles. The speed with which vacuolation occurs indicates the need for rapid techniques of fixation in histological studies of these cells. Any vital staining procedures, for example the McGovern silver method used by Vrabec (1958) or the alizerine red technique used by Chi and others (1958) for staining cement substance, must be assessed in the light of the extensive post-mortem changes which these techniques inevitably produce. Physiological studies of the cornea employing excised eyes (Langham and Taylor, 1956; Harris, 1957) should also be re-assessed, because it is very unlikely that endothelial cells would function normally in vitro if the cytoplasm were grossly vacuolated. Our studies have shown how very difficult it is to prevent this degenerative change. They also indicate how readily the corneal endothelium may be injured by solutions or instruments introduced into the anterior chamber in the course of surgical procedures, and they emphasize the need for extreme care in handling this tissue, particularly in corneal grafting. 
Several recent papers (Flocks, 1958; Kornzweig, Feldstein, and Schneider, 1958) describe pathological changes in the trabecular meshwork in cases of chronic simple glaucoma. In reports by Teng, Paton, and Katzin (1955) and Teng, Katzin, and Chi (1957), which have been widely quoted, degenerative changes are described in the region of Schlemm's canal. Because their material was stored in an eye bank for an unstated period of time, it is impossible to avoid the conclusion that gross post-mortem changes were present in the filtration angles of these and other eyes described in the literature where the interval between enucleation and fixation has been prolonged (Ashton and Speakman, 1958). Perfusion experiments on excised eyes are also open to criticism because of the degenerative changes which occur in the cells lining the drainage channels as soon as an eye is removed from its normal environment.

\section{Summary}

The endothelial cells lining the posterior surface of the cornea and the trabecular wall of Schlemm's canal develop numerous large vacuoles unless special fixation techniques are employed to prevent post-mortem change. The morphological similarity of the degenerative changes displayed by these cells and the rapidity of their onset suggest that the endothelial cells in these two regions may also have important functional similarities. It follows, therefore, that an intact layer of endothelium (lining the inner wall of the canal) may be essential for maintaining a normal resistance to aqueous outflow through the narrow, attenuated drainage channels near the canal wall by preventing swelling of the trabecular fibres in this region.

The findings indicate some of the difficulties in interpreting histological preparations of the corneal endothelium and trabecular meshwork which have not been rapidly fixed, and in assessing the significance of in vitro experiments on the excised eye which concern the normal functioning of these cells.

I am greatly indebted to Prof. Norman Ashton for many helpful suggestions during the preparation of this paper and to Mr. B. Fiddy for his valuable technical assistance.

\section{REFERENCES}

Ashton, N., and Speakman, J. S. (1958). Proc. roy. Soc. Med. (In press.)

Chi, H. H., Teng, C. C., and Katzin, H. M. (1958). Amer. J. Ophthal., 45, 518.

Flocks, M. (1958). Trans. Amer. Acad. Ophthal. Otolaryng., $62,556$.

Garron, L. K., Feeney, M. L., Hogan, M. J., and McEwen, W. K. (1958). Amer. J. Ophthal., 46, No. 1, Part 2, p. 27.

HARRIS, J. E. (1957). Ibid., 44, No. 5, Part 2, p. 262.

KoRnZWeig, A. L., FeldSTEIn, M., and SchneIDER, J. (1958). Ibid., 46, No. 3, Part 1, 311.

LANGHAM, M. E., and TAYLOR, I. S. (1956). Brit. J. Ophthal., 40, 321.

Nuel, J. P., and CORNIL, F. (1890). Arch. Ophtal. (Paris), 10, 309.

Speakman, J. S. (1959). Brit. J. Ophthal., 43, 129.

Teng, C. C., Katzin, H. M., and ChI, H. H. (1957). Amer. J. Ophthal., 43, 193.

- Paton, R. T., and Katzin, H. M. (1955). Ibid., 40, 619.

VRABEC, F. (1958). Brit. J. Ophthal., 42, 529.

Wolter, J. R. (1957). Klin. Mbl. Augenheilk., 130, 666. 